\title{
Plan estratégico para el desarrollo del eje de vinculación con la sociedad, Unemi, Ecuador
}

\section{A Strategic Plan to Develop the Community Outreach Hub at UNEMI, Ecuador}

Eduardo Espinoza-Solís Universidad Estatal de Milagro Ecuador

eespinozas@unemi.edu.ec

iD https://orcid.org/0000-0001-8007-8227

Norberto Murillo-Dávila

Universidad Estatal de Milagro

Ecuador

iD https://orcid.org/0000-0003-2721-5661

Cecilia Solís-Ríos

Universidad Estatal de Milagro

Ecuador

csolisr3@unemi.edu.ec

D. https://orcid.org/0000-0002-1433-3530

Verónica Sandoval-Tamayo

Universidad Estatal de Milagro

Ecuador

vsandovalt@unemi.edu.ec https:/orcid.org/0000-0002-3695-8486

Andrea Velásquez Universidad Estatal de Milagro

Ecuador andreavelasquezb1@gmail.com

Recibido: 29/05/2020 • Aceptado:04/09/2020

Resumen. La Universidad Estatal de Milagro (Unemi) se constituye como una institución de educación superior clave en el desarrollo de la región 5 del Ecuador, es por ello que se ha desarrollado un plan estratégico de vinculación con la sociedad, que interrelaciona todas las carreras que posee la universidad y las funciones sustantivas de docencia e investigación. La metodología se ejecuta a través de una planificación fundamentada en los dominios de la universidad, las líneas de investigación que 
Revista Universidad en DiÁlogo • Vol. 11, N. 1, Enero-Junio, 2021 • 37-60

ISSN 2215-2849 • EISSN: 2215-4752

URL: http://www.revistas.una.ac.cr/index.php/dialogo/index CorReo ElECTRÓNICO: universidadendialogo@una.cr

DOI: http://doi.org/10.15359/udre.11-1.2

promueven la vinculación, los objetivos de desarrollo internacionales, nacionales y locales con los que se crearon programas y proyectos que permiten incrementar el impacto y la cobertura en el territorio, así como la difusión de los resultados obtenidos. El plan presenta estrategias de alianzas con comunidades para el desarrollo de acciones conjuntas, apoyo del sector privado para incrementar recursos, mismos que en el sector público son limitados, y alianzas con otras universidades de la región para el desarrollo de proyectos mancomunados que faciliten el campo de acción de beneficiarios, a través de una sinergia de acción masiva.

Palabras clave: acción social, desarrollo sostenible, extensión universitaria, tercera misión.

\begin{abstract}
The Universidad Estatal de Milagro (UNEMI) is a key higher education institution in the development of region 5 in Ecuador. Therefore, a community outreach strategic plan has been developed; it interrelates all university's careers and the teaching and research substantive functions. The methodology is executed through a plan based on the university's domains, the research lines that promote the outreach, the international, national, and local development objectives with which programs and projects were created to increase the impact and coverage in the territory, and the dissemination of the results obtained. As a result, the plan offers strategies of alliances with communities to develop joint actions, support from the private sector to increase resources, which are limited in the public sector, and alliances with other universities in the region to develop joint projects to facilitate the beneficiaries' field of action through a synergy of massive action.
\end{abstract}

Keywords: social action, sustainable development, third mission, university extension.

\title{
Introducción
}

Dentro de sus funciones, la universidad ecuatoriana ha definido la vinculación con la sociedad como una de las tareas primordiales, con el objeto de aportar en la solución de los problemas fundamentales de la sociedad, interrelacionando los procesos propios de la institución de educación superior con la dinámica de las organizaciones sociales, instituciones y comunidad en general.

La actividad de vinculación con la colectividad ha generado un nexo directo en el intercambio de conocimientos y prácticas en las diferentes organizaciones, barrios y comunidades del país, con el objeto de compartir con quienes más necesitan, dado que la vinculación con la sociedad hace referencia a los "programas de educación continua, gestión de redes, cooperación y desarrollo, relaciones internacionales, difusión y distribución del saber que permitan la democratización del conocimiento y el desarrollo de la sociedad" (CES, 2017). 
En la proyección de la vinculación con la sociedad hay que tener presente que esta no se refiere únicamente a un trabajo entre estudiantes y docentes, se trata de vincular a toda la población, deben interrelacionarse tanto la comunidad interna como externa, dando como resultado las relaciones sociales que se dan entre los sujetos participantes (diagnóstico).

Está dirigido de un modo sistémico y eficiente a la promoción de la cultura y del desarrollo para la comunidad (objetivo), para coadyuvar a la solución de la problemática social (problema) y contribuir al desarrollo de la comunidad, mediante la apropiación de la cultura que ha acumulado la sociedad en su desarrollo (contenido), a través de la participación activa de la comunidad (método), planificada en el tiempo y observando ciertas estructuras organizativas (formas), con ayuda de ciertos objetos (medios) e instrumentando indicadores que le permitan medir la calidad (evaluación) (Reuvic, 2012).

De acuerdo con las normas ecuatorianas vigentes, se deberá contar con modelos que permitan vincular las funciones sustantivas de la educación superior, por ello la Universidad Estatal de Milagro se ha planteado dentro de su Plan Estratégico de Desarrollo Institucional el objetivo general de vinculación para el año 2021: "Mejorar la pertinencia, calidad e impacto de los programas y proyectos de Vinculación con la Sociedad en función del desarrollo regional y los desafíos de la UNEMI" (Unemi, 2018a). Este documento constituye un modelo general que permitirá a las facultades integrar los aspectos disciplinares y profesionales que se desarrollan dentro de cada una, para así generar proyectos que respondan a las políticas y estrategias de vinculación institucional.

De allí que se pretende, a partir de los planes operativos anuales, gestionar el macro objetivo año a año.

\section{Marco teórico}

De acuerdo con D'Este, Castro-Martínez y Molas-Gallart (2009), la vinculación con la colectividad o la sociedad es un conjunto de acciones universitarias que se relaciona con a) generar conocimiento y capacidades en colaboración con organizaciones y agentes no académicos y b) aplicabilidad del conocimiento y otras capacidades existentes en la universidad, fuera del entorno académico, con el resto de la sociedad.

Para ejecutar esta función sustantiva del entorno universitario, un plan es el parámetro técnico-político dentro del cual se enmarcan los programas y proyectos, constituyéndose en un elemento emergente, producto de la interacción 
Revista Universidad en DiÁlogo • Vol. 11, N. 1, Enero-Junio, 2021 • 37-60

ISSN 2215-2849 • EISSN: 2215-4752

URL: http://www.revistas.una.ac.cr/index.php/dialogo/index CorReo ElECTRÓNICO: universidadendialogo@una.cr DOI: http://doi.org/10.15359/udre.11-1.2

entre sus partes. Es un conjunto organizado, coherente e integrado de actividades, servicios o procesos, expresados en un conjunto de proyectos relacionados o coordinados entre sí y que son de similar naturaleza. Un programa está constituido por una constelación o conjunto de proyectos (Ander-Egg, 2013).

Un proyecto de vinculación con la sociedad es el conjunto de actividades que la institución desarrolla, en el marco de un programa, en un sector y tiempo determinados, para propiciar la solución de un problema, de una necesidad insatisfecha, considerada como relevante y prioritaria para el desarrollo de un determinado grupo humano, localidad o región (Garrocho y Segura, 2012).

Los proyectos de vinculación de la Unemi dan respuesta a las diversas demandas sociales: problemas de la sociedad, la actualización del perfil profesional, los requerimientos del mercado ocupacional y la proyección del propio proyecto universitario. Para ello se plantea realizar actividades en torno a proyectos y eventos de vinculación: académicos, productivos, sociales, deportivos, culturales, artísticos y tecnológicos, seguimiento y acompañamiento a graduados y graduadas, prácticas pre-profesionales y pasantías.

Los proyectos institucionales de vinculación con la sociedad se desarrollarán en un marco de investigación-acción-participación en el cual los y las estudiantes aplicarán competencias teórico-prácticas relacionadas con su carrera, culminando con un informe que evidencie las actividades realizadas durante el proceso.

La Zona 5 comprende los territorios de las provincias de Santa Elena, Los Ríos, Bolívar, Galápagos y Guayas, excepto los cantones de la Zona 8: Guayaquil, Durán y Samborondón.

La población proyectada al 2017 de esta zona es de 2678 261, 15,96\% de la población del Ecuador (INEC, 2012).

Las ciudades más pobladas de la zona son: Milagro, Quevedo, Babahoyo, Santa Elena y Daule, con poblaciones entre 120000 y 174000 habitantes. Seguidas de Naranjal, Vinces, El Empalme, Guaranda y La Libertad, con poblaciones entre 69000 y 96000 habitantes (INEC, 2018).

En lo relacionado con la Administración pública, de acuerdo con la micro planificación vigente desde el año 2012, en el territorio zonal funcionan 25 distritos y 193 circuitos administrativos para la prestación de servicios de los ministerios del Interior, Justicia, Salud, Educación, MIES, Deporte y la Secretaría de Gestión de Riesgo. 


\section{Prioridades de la Zona 5}

Por su parte, la Agenda Zonal 5 prioriza las problemáticas territoriales de la zona, con base en las cuales se han identificado las metas nacionales establecidas en el Plan Nacional de Desarrollo. Los problemas priorizados son los siguientes:

- Deficiente desarrollo del sector productivo rural.

- Persistencia de pobreza y extrema pobreza.

- Deficiente cobertura y atención de servicios sociales de salud, educación y cuidados de la primera infancia.

- Inseguridad ciudadana.

- Desequilibrado desarrollo territorial: sector rural deficientemente atendido.

- Déficit de programas de vivienda social, cobertura de servicios básicos y conectividad.

- Contaminación ambiental y deterioro de recursos naturales. Énfasis en fuentes hídricas, mares y reducción de la cobertura natural.

\section{Análisis de detección de necesidades locales}

Dentro de la relación investigación-vinculación se han realizado estudios para determinar las necesidades prioritarias de la población vinculada con la universidad (Garzon-Castrillón y Zúñiga, 2017). A continuación, los datos más relevantes:

- El 80\% de las parroquias estudiadas tiene una población inferior a 20 000 habitantes y el 20\% (Naranjal y Yaguachi) tiene una población superior a los 50000 habitantes.

- La principal actividad económica es la agricultura, adicionalmente en una parroquia se destaca el comercio y la ganadería, en cuatro parroquias el turismo y en dos la pesca artesanal.

- Se afirma que en general la fortaleza de las parroquias estudiadas está en su gente: se caracteriza por ser humilde, trabajadora, hospitalaria, amable, respetuosa, colaboradora, emprendedora y solidaria. 
- Dos parroquias no tienen cobertura total de servicios básicos.

- En las diez parroquias existen organizaciones sociales sin ánimo de lucro y privadas, entre las que se pueden destacar grupos culturales, grupos de bienestar de personas con discapacidad, grupos de la tercera edad, fundaciones sociales y para apoyar el arte, defensores del medio ambiente, grupos folclóricos, brigadas de seguridad ciudadana, grupos culturales y asociaciones de las principales actividades económicas: comercio, taxis, transporte, mercado, cangrejos, agricultura, pesca, artesanía, ganadería y motociclistas independientes, entre otras.

\section{Necesidades percibidas}

Los resultados se agrupan en tres áreas de necesidades, estas áreas serán posteriormente las áreas de intervención de la Unemi por medio de proyectos de vinculación con la sociedad:

- Saludy bienestar de la población: Recoge las necesidades relacionadas con problemas de salud física y psicológica, y problemas locales que afectan la salud.

- Educación y comunicación: Necesidades relacionadas con la educación, las dificultades en la comunicación social y la cultura.

- Desarrollo empresarial y productivo: Comprende las necesidades relacionadas con la gestión y la tecnificación de las empresas privadas y sociales, y las del sector productivo (agricultura y pesca).

En general, las necesidades prioritarias identificadas por los y las informantes en las parroquias objeto de la investigación son las siguientes:

\section{Área de salud y bienestar de la población:}

- Consumo de drogas en niños, niñas y adolescentes.

- Embarazos a temprana edad.

- Déficit en la atención en salud. 
URL: http://www.revistas.una.ac.cr/index.php/dialogo/index

CORREO ELECTRÓNICO: universidadendialogo@una.cr

DOI: http://doi.org/10.15359/udre.11-1.2

\section{Área de educación y comunicación:}

- Niños, niñas y adolescentes abandonan el colegio.

- Personas adultas mayores que no saben leer ni escribir.

- No existen programas de comunicación social.

\section{Área de desarrollo empresarial y productivo:}

- No existe promoción turística en la zona.

- Bajo conocimiento para proyectos de emprendimiento y falta de apoyo.

- Falta ayuda técnica para mejorar los cultivos.

\section{Metodología}

Para ejecutar el objetivo de vinculación con la sociedad, la Unemi, a través de su Departamento de Extensión Universitaria, plantea la creación de un Plan Estratégico de Vinculación para el 2021, en el que se incluyen los siguientes lineamientos:

1. Todos los programas se enfocarán en el desarrollo regional (desde el barrio o comunidad hasta la región 5 como foco principal, considerando actuar en otras regiones de acuerdo con la pertinencia).

2. Los programas institucionales que integren a los proyectos de carrera y a los proyectos de vinculación se articularán a partir de los siguientes elementos:

a. Los ODS y el desarrollo regional.

b. Los objetivos del PND y el desarrollo regional.

c. Los GAD, el sector público y el desarrollo regional.

d. El sector privado, la economía popular y solidaria y el desarrollo regional.

e. La educación continua y el desarrollo regional. 
3. Cada carrera ejecutará cada año al menos dos proyectos, los cuales involucrarán a más de dos carreras.

4. A cada docente titular u ocasional que participe en un proyecto de vinculación se le debe asignar un rango de 64-80 horas semestre, con cargo a docencia o investigación.

5. La Unemi asignará los recursos necesarios para financiar los proyectos de vinculación. La asignación será incremental hasta llegar al 1\% del presupuesto institucional en el 2021.

6. La Unemi liderará, al menos, un proyecto regional a través de redes universitarias.

7. La coordinación de los programas y proyectos de vinculación a nivel institucional le corresponderá al Departamento de Extensión Universitaria.

8. Los programas y proyectos serán evaluados, en especial productos, resultados e impactos en los beneficiarios, así como la participación de profesores, profesoras y estudiantes.

9. Cada carrera ofertará al menos un curso de Educación Continua Avanzada (Reglamento Régimen Académico, 2017).

10.El seguimiento, a nivel institucional, de los graduados y las graduadas, en lo gerencial, es responsabilidad del Departamento de Extensión Universitaria y, en lo operativo, del Área de Gestión Técnica Académica.

11. A nivel institucional, las prácticas pre-profesionales, en lo gerencial, son responsabilidad del Departamento de Extensión Universitaria y, en lo operativo, del Área de Gestión Técnica Académica.

12. Cada proyecto de investigación incluirálos mecanismos de transferencia de conocimientos para la vinculación de acuerdo con su pertinencia.

13.La Unemi otorgará reconocimientos específicos e incentivos a profesores y profesoras que se destaquen en sus proyectos de vinculación.

14. A nivel institucional, el proyecto Unemi-Empleo, en lo gerencial, es responsabilidad del Departamento de Extensión Universitaria y, en lo operativo, del Área de Gestión Técnica Académica. 
URL: http://www.revistas.una.ac.cr/index.php/dialogo/index

CORREO ELECTRÓNICO: universidadendialogo@una.cr

DOI: http://doi.org/10.15359/udre.11-1.2

\section{Resultados}

\section{Figura 1}

Mapa Estratégico Eje Vinculación con la Sociedad, Unemi al 2021

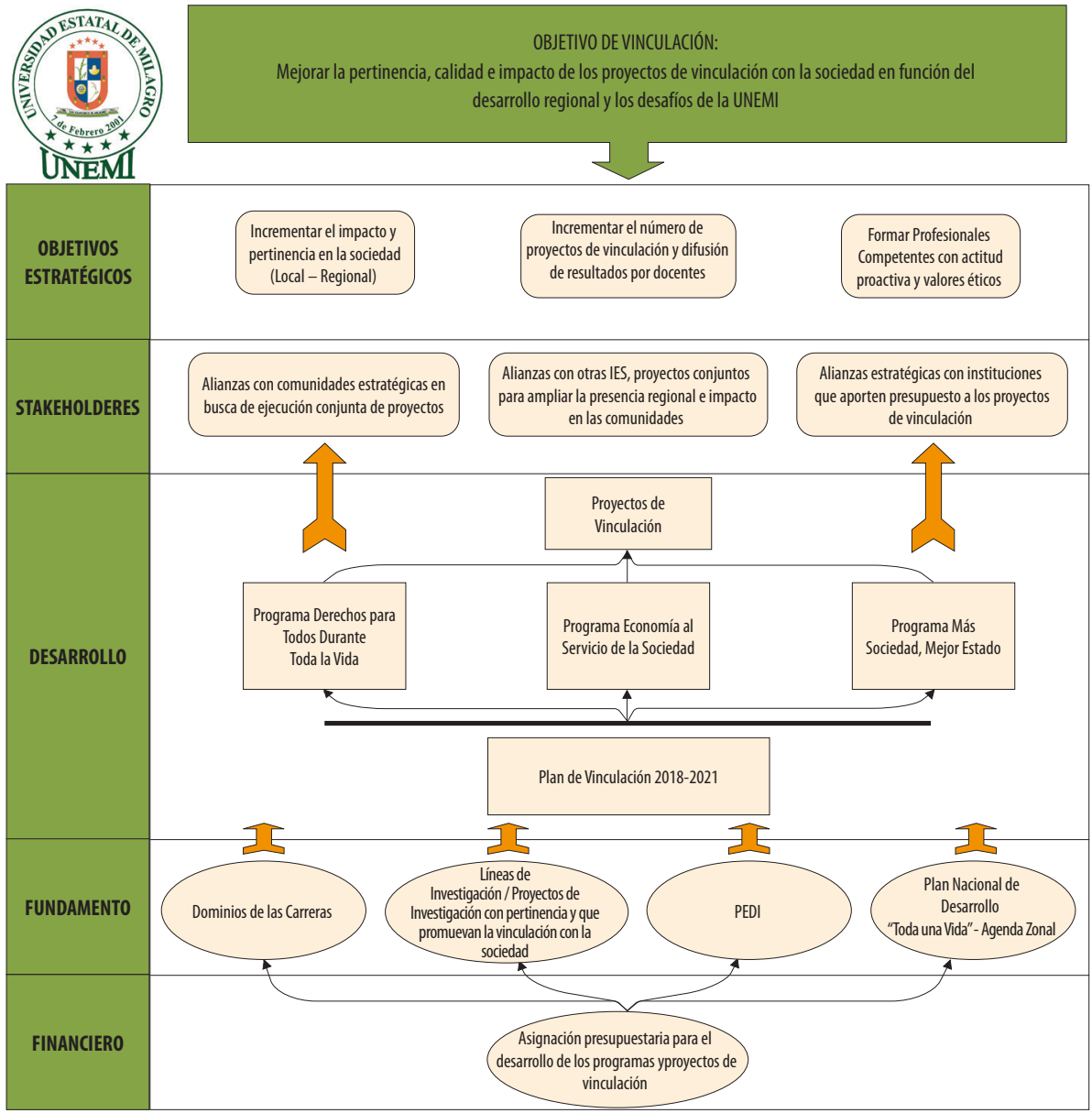

Nota. Plan Estratégico de Vinculación, Unemi

Como resultado del trabajo y de los lineamientos establecidos en la metodología, se realizó un Plan Estratégica al 2021, como se observa en la Figura 1. Se creó un mapa estratégico en el que se establece en la base la necesidad de asignaciones presupuestarias para la ejecución de los programas y proyectos de vinculación, los 
cuales se fundamentan en el dominio de las carreras, las líneas y los proyectos de investigación que promuevan la vinculación entre el Plan Estratégico de Desarrollo Institucional, el Plan Nacional de Desarrollo y la Agenda Zonal 5.

Se crearon tres programas de vinculación a tono con los tres ejes del Plan Nacional de Desarrollo (Toda una Vida) del Gobierno Nacional. Se considera necesaria la presencia de stakeholders en las comunidades para facilitar la ejecución de los proyectos en territorio con otras instituciones de educación superior, para ampliar la cobertura y presencia regional y el impacto en las comunidades, y con otras instituciones en busca de fondos externos que aporten al financiamiento de los programas y proyectos (Unemi, 2018).

En el del Plan Estratégico se plantean también los siguientes elementos orientadores:

\section{Visión:}

"Referente de la vinculación con la sociedad en la región, aportando a la UNEMI a ser una universidad de docencia e investigación"

\section{Misión:}

"Somos una unidad estratégica de la Universidad Estatal de Milagro encargada de cumplir con los objetivos de vinculación con la sociedad, bolsa de empleo y emprendimiento, facilitando las propuestas de las diferentes facultades y carreras para el desarrollo efectivo de programas y proyectos que permitan la transferencia de conocimiento y tecnología, contribuyendo al desarrollo sustentable local, regional y nacional".

Así también, se plantean los siguientes objetivos operativos:

\section{Objetivos operativos en proyectos de vinculación (PV)}

Objetivo operativo n..$^{\circ}$ 1: Implementar el Plan de Vinculación con la Sociedad

\section{Acciones:}

1. Crear programas de vinculación con la sociedad, cuatrienales, acordes con los objetivos de desarrollo sostenible, los objetivos del Plan Nacional de Desarrollo y de la Agenda Zonal, las líneas de investigación y los informes de resultados de proyectos de investigación institucionales. 
2. Apoyar a las facultades en la presentación de proyectos de vinculación con la sociedad que aborden los temas de interés de la Unemi y la región, dentro de los objetivos y del marco establecidos.

3. Acompañar a las facultades para la presentación de proyectos transversales entre carreras.

\section{Tabla 1}

Medios de verificación del objetivo operativo $P V n .{ }^{\circ} 1$

\begin{tabular}{clc}
\hline \multicolumn{1}{c}{ Indicador } & \multicolumn{1}{c}{ Actores } & Meta \\
\hline Cumplimiento del Plan de Vinculación & Extensión Universitaria & $100 \%$ \\
& Facultades & \\
& Investigación & \\
\hline
\end{tabular}

Nota. Elaboración propia

Dentro de los medios de verificación se encuentra como indicador el cumplimiento del $100 \%$ del plan por parte de los actores involucrados, como se observa en la Tabla 1.

Objetivo operativo n. ${ }^{\circ}$ 2: Consolidar programas y proyectos de vinculación con la sociedad que respondan a las necesidades del entorno.

\section{Acciones:}

1. Desarrollar convocatorias anuales dirigidas a la comunidad universitaria para la presentación de proyectos de vinculación

2. Revisar y brindar acompañamiento a las facultades para la aprobación de los proyectos y gestionar la respectiva asignación presupuestaria

3. Monitorear la ejecución de los proyectos de vinculación y la emisión de informes parciales y finales de avances de los proyectos

4. Realizar anualmente el seguimiento y la actualización de avances de los programas de vinculación

5. Realizar un informe parcial de avances en los impactos 


\section{Tabla 2}

Medios de verificación del objetivo operativo $P V n .{ }^{\circ} 2$

\begin{tabular}{cll}
\hline \multicolumn{1}{c}{ Indicador } & \multicolumn{1}{c}{ Actores } & Meta \\
\hline Cumplimiento de avances de programas de vinculación & Comisión de Vinculación & $100 \%$ \\
Cumplimiento de ejecución de proyectos de vinculación & Extensión Universitaria & \\
& Facultades & \\
& &
\end{tabular}

Nota. Elaboración propia

Dentro de los medios de verificación se encuentran como indicadores el cumplimiento del $100 \%$ de los avances en programas y la ejecución de proyectos de vinculación por parte de los actores involucrados, como se observa en la Tabla 2.

Objetivo operativo n. ${ }^{\circ}$ 3: Sistematizar los procesos de vinculación con la sociedad

\section{Acciones:}

1. Generar un cronograma de trabajo conjunto con el Departamento de Tecnologías de la Información (TIC).

2. Desarrollar los módulos para registro de programas y proyectos y el respectivo seguimiento de todas las partes involucradas.

3. Socializar la herramienta una vez que esté lista.

\section{Tabla 3}

Medios de verificación del objetivo operativo $P V n{ }^{\circ} 3$

\begin{tabular}{lll}
\multicolumn{1}{c}{ Indicador } & \multicolumn{1}{c}{ Actores } & \multicolumn{1}{c}{ Meta } \\
\hline Herramienta informática activa & Extensión Universitaria & $100 \%$ \\
Socialización & TIC & 1 \\
\hline
\end{tabular}

Nota. Elaboración propia

Como indicadores, dentro de los medios de verificación, se encuentran el cumplimiento del $100 \%$ de la creación de la herramienta informática para el desarrollo de la vinculación, tanto para docentes y personal administrativo como para estudiantes, y su respectiva socialización por parte de los actores involucrados, como se observa en la Tabla 3. 
Objetivo operativo n. ${ }^{\circ}$ 4: Ejecutar de manera conjunta proyectos de vinculación con otras IES

\section{Acciones:}

1. Establecer, por medio de convenios, alianzas estratégicas con Instituciones de Educación Superior (IES) nacionales e internacionales, a través de la red de la Unión Latinoamericana de Extensión Universitaria (ULEU), a la que pertenece la Unemi, y así desarrollar proyectos conjuntos.

2. Realizar un proyecto anual (desde el 2019) de manera conjunta con otra IES de la regi

3. Liderar hasta el 2021 un proyecto de vinculación regional en el que participen otras IES.

\section{Tabla 4}

Medios de verificación del objetivo operativo PVn ${ }^{\circ} 4$

\begin{tabular}{llc}
\hline \multicolumn{1}{c}{ Indicador } & \multicolumn{1}{c}{ Actores } & Meta 2019 \\
\hline Número de convenios con otras IES & Extensión Universitaria & $100 \%$ \\
Cumplimiento del proyecto anual conjunto & $\begin{array}{l}\text { Relaciones Interinstitucionales } \\
\text { Otras IES }\end{array}$ & 1 \\
Cumplimiento del proyecto regional & ULEU & 1 \\
\hline
\end{tabular}

Nota. Elaboración propia

Dentro de los medios de verificación se encuentran como indicadores el cumplimiento del $100 \%$ de los convenios establecidos a través del área de relaciones interinstitucionales, el desarrollo de un proyecto conjunto anual y el liderazgo de un proyecto regional por parte de los actores involucrados, como se observa en la Tabla 4.

Objetivo operativo n. ${ }^{\circ}$ 5: Realizar eventos de divulgación de resultados de la vinculación con la sociedad

\section{Acciones:}

1. Elaborar una feria anual de vinculación (desde el 2019

2. Presentación de artículos científicos con base en las experiencias y el desarrollo de la vinculación al 201 
3. Elaboración del Congreso de Vinculación con la Sociedad para inicios del año 20

4. Participar en eventos como congresos y jornadas de vinculación a nivel nacional e internacional

Tabla 5

Medios de verificación del objetivo operativo $P V n .{ }^{\circ} 5$

\begin{tabular}{llc}
\hline \multicolumn{1}{c}{ Indicador } & \multicolumn{1}{c}{ Actores } & Meta \\
\hline Cumplimiento de elaboración de la feria anual & Vicerrectorado Académic & 1 \\
Número de artículos a partir de la vinculación & $\begin{array}{l}\text { Extensión Universitaria } \\
\text { Facultades }\end{array}$ & 5 \\
Cumplimiento de elaboración del congreso & $\begin{array}{l}\text { Relaciones Públicas } \\
\text { Investigación }\end{array}$ & 1 \\
\hline
\end{tabular}

Nota. Elaboración propia

Entre los medios de verificación se encuentran como indicadores el cumplimiento de elaborar una feria anual de exposición de los proyectos de vinculación ejecutados, cinco artículos científicos a partir de las experiencias de vinculación y un congreso por parte de los actores involucrados, tal como se observa en la Tabla 5.

Objetivo operativo $\mathbf{n} .^{\circ}$ 6: Realizar alianzas estratégicas con instituciones y empresas en busca de apoyo financiero para la ejecución de los proyectos

\section{Acciones:}

1. Búsqueda y gestión de empresas, instituciones y organizaciones sin fines de lucro (ONG) que puedan cofinanciar los proyectos de vinculación.

2. Firmas de convenios y acuerdos para apoyo financiero.

\section{Tabla 6}

Medios de verificación del objetivo operativo $P V n{ }^{\circ} 6$

\begin{tabular}{clc}
\hline Indicador & \multicolumn{1}{c}{ Actores } & Meta \\
\hline Número de convenios firmados & $\begin{array}{l}\text { Extensión Universitaria } \\
\text { Relaciones Interinstitucionales }\end{array}$ & 5 \\
\hline
\end{tabular}

Nota. Elaboración propia 
Dentro de los medios de verificación se encuentra como indicador el cumplimiento de firmar cinco convenios para recibir apoyo financiero en el desarrollo de las actividades de vinculación por parte de los actores involucrados, como se observa en la Tabla 6.

\section{Objetivo operativo n. ${ }^{\circ}$ 7: Crear el Voluntariado Unemi}

\section{Acciones:}

1. Crear legalmente el voluntaria.

2. A partir del voluntariado, buscar apoyo externo para la ejecución de los proyecto.

3. Incentivar a la comunidad para que se involucre en las actividades de labor comunitaria.

\section{Tabla 7}

Medios de verificación del objetivo operativo $P V n .{ }^{\circ} 7$

\begin{tabular}{clc}
\hline Indicador & \multicolumn{1}{c}{ Actores } & Meta \\
\hline Creación del Voluntariado & $\begin{array}{l}\text { Extensión Universitaria } \\
\text { Relaciones Interinstitucionales }\end{array}$ & 1 \\
\hline
\end{tabular}

Nota. Elaboración propia

En los medios de verificación se encuentra como indicador el cumplimiento de la creación de un voluntariado para el desarrollo de la vinculación por parte de los actores involucrados, como se observa en la Tabla 7.

\section{Objetivos operativos de prácticas pre profesionales (PPP)}

Objetivo operativo n. ${ }^{\circ}$ : Ubicar a los y las estudiantes en el proceso de prácticas pre profesionales

\section{Acciones:}

1. Alimentar el sistema de prácticas con las asignaciones por itinerario.

2. Ubicar a los y las estudiantes en las empresas.

3. Asignar una persona supervisora / tutor/a académico/a.

4. Realizar un informe de resultados por período académico. 


\section{Tabla 8}

Medios de verificación del objetivo operativo PPP $n .^{\circ} 1$

\begin{tabular}{clc}
\hline \multicolumn{1}{c}{ Indicador } & \multicolumn{1}{c}{ Actores } & Meta \\
\hline Estudiantes realizando prácticas & Secretaría de Gestión Técnica Académica & $100 \%$ \\
& Extensión Universitaria & \\
& Relaciones Interinstitucionales & \\
& Facultades & \\
\hline
\end{tabular}

Nota. Elaboración propia

Entre los medios de verificación se encuentra como indicador el cumplimiento del $100 \%$ de los itinerarios de prácticas pre profesionales realizados por los y las estudiantes, por parte de los actores involucrados, como se observa en la Tabla 8.

Objetivo operativo n. ${ }^{\circ}$ 2: Crear un portafolio de productos por carrera

\section{Acciones:}

1. Establecer perfiles por carreras e itinerarios.

2. Crear el portafolio de practicantes / pasantes Unemi.

3. Socializar el portafolio con la comunidad.

Tabla 9

Medios de verificación del objetivo operativo PPP $n .^{\circ} 2$

\begin{tabular}{rlc}
\hline \multicolumn{1}{c}{ Indicador } & \multicolumn{1}{c}{ Actores } & Meta \\
\hline Creación del portafolio & SGTA & 1 \\
& Extensión Universitaria & \\
& Relaciones Interinstitucionales & \\
& Facultades & \\
\hline
\end{tabular}

Nota. Elaboración propia

Dentro de los medios de verificación se encuentra la creación de un portafolio, por parte de los actores involucrados, que agrupe a todas las carreras para el desarrollo de las prácticas profesionales, como se observa en la Tabla 9. 
Objetivo operativo n. ${ }^{\circ}$ : Generar una mejora continua en el proceso de prácticas pre profesionales

\section{Acciones:}

1. Revisión anual de los procesos.

2. Establecer un proceso de pre-inscripción de prácticas para agilizar el proceso.

3. Generar un proceso mejorado.

4. Socializar procesos.

Tabla 10

Medios de verificación del objetivo operativo PPP $n .{ }^{\circ} 3$

\begin{tabular}{cll}
\hline \multicolumn{1}{c}{ Indicador } & \multicolumn{1}{c}{ Actores } & \multicolumn{1}{c}{ Meta } \\
\hline Proceso mejorado de manera continua & SGTA & 1 Anual \\
& Extensión Universitaria & \\
& Aseguramiento de la Calidad & \\
& Facultades & \\
\hline
\end{tabular}

Nota. Elaboración propia

Dentro de los medios de verificación se encuentra el cumplimiento una vez al año de un proceso de mejora continua, utilizando la retroalimentación obtenida en cada período de prácticas por parte de los actores involucrados, como se observa en la Tabla 10.

Objetivo operativo n. ${ }^{\circ}$ 4: Establecer un proceso de formación previa para la realización de las prácticas pre profesionales

\section{Acciones:}

1. Socializar la metodología de las prácticas previo a la vinculación estudiantes-empresa.

2. Realizar charlas psicológicas y motivacionales para apoyar la vinculación a las empresas de manera satisfactoria. 


\section{Tabla 11}

Medios de verificación del objetivo operativo PPP $n .^{\circ} 4$

\begin{tabular}{lll}
\hline \multicolumn{1}{c}{ Indicador } & \multicolumn{1}{c}{ Actores } & \multicolumn{1}{c}{ Meta } \\
\hline Realización de evento de & SGTA & Dos por año \\
socialización & Extensión Universitaria & \\
& Bienestar Universitario & \\
& Empresas invitadas a participar & \\
\hline
\end{tabular}

Nota. Elaboración propia

Entre los medios de verificación se encuentra la realización de dos eventos de socialización, tanto para docentes y personal administrativo como para estudiantes, por parte de los actores involucrados, como se observa en la Tabla 11.

Objetivo operativo n. ${ }^{\circ}$ 5: Potenciar la inclusión de los y las estudiantes en las principales empresas de la zona en el proceso de prácticas pre profesionales

\section{Acciones:}

1. Solicitar a Relaciones Interinstitucionales la gestión con empresas representativas de la zona.

2. Vincular a los y las estudiantes de acuerdo con sus perfiles en las empresas representativas.

3. Establecer un proceso de retroalimentación personalizado con las empresas para incrementar la recepción de estudiantes.

\section{Tabla 12}

Medios de verificación del objetivo operativo PPP $n .^{\circ} 5$

\begin{tabular}{lll}
\hline \multicolumn{1}{c}{ Indicador } & \multicolumn{1}{c}{ Actores } & Meta \\
\hline Estudiantes vinculados/as con las & SGTA & $10 \%$ \\
empresas más representativas de la zona & Extensión Universitaria & \\
& Bienestar Universitario \\
& Empresas invitadas a participar & \\
\hline
\end{tabular}

Nota. Elaboración propia 
Dentro de los medios de verificación, el 10\% de los y las estudiantes debe estar vinculado con sus procesos de prácticas en las empresas más representativas de la zona, por parte de los actores involucrados, como se observa en la Tabla 12.

\section{Objetivos operativos de seguimiento a graduados y graduadas (SG)}

Objetivo operativo n. ${ }^{\circ}$ 1: Interactuar con las personas graduadas de la Unemi Acciones:

1. Encuestar a las personas graduadas.

2. Conocer la situación de las personas graduadas.

3. Realizar jornadas de conferencias periódicas.

4. Realizar integración deportiva.

5. Realizar un informe de resultados anual.

Tabla 13

Medios de verificación del objetivo operativo $S G n .^{\circ} 1$

\begin{tabular}{clc}
\hline \multicolumn{1}{c}{ Indicador } & \multicolumn{1}{c}{ Actores } & Meta \\
\hline Interacción de personas graduadas con la Unemi & $\begin{array}{l}\text { SGTA } \\
\text { Extensión Universitaria }\end{array}$ & $30 \%$ \\
& \multicolumn{2}{l}{$\begin{array}{l}30 \% \\
\text { E }\end{array}$} \\
\hline
\end{tabular}

Nota. Elaboración propia

Entre los medios de verificación se encuentra como indicador que el 30\% de las personas graduadas debe realizar alguna interacción con la universidad por medio de los actores involucrados, como se observa en la Tabla 13.

Objetivo operativo n. ${ }^{\circ}$ 2: Generar una mejora continua del proceso de seguimiento de graduados y graduadas

Acciones:

1. Revisión anual de los procesos - cuestionarios

2. Generar un proceso mejorado

3. Sistematizar el proceso

4. Socializar procesos 


\section{Tabla 14}

Medios de verificación del objetivo operativo $S G n .^{\circ} 2$

\begin{tabular}{cll}
\hline \multicolumn{1}{c}{ Indicador } & \multicolumn{1}{c}{ Actores } & \multicolumn{1}{c}{ Meta } \\
\hline Proceso mejorado de manera continua & SGTA & 1 Anual \\
& Extensión Universitaria & \\
& Aseguramiento de la Calidad & \\
& TIC & \\
\hline
\end{tabular}

Nota. Elaboración propia

Dentro de los medios de verificación se encuentra como indicador el cumplimiento de un proceso de mejora continua anual por parte de los actores involucrados, como se observa en la Tabla 14.

Objetivo operativo n. ${ }^{\circ}$ 3: Desarrollar un portafolio de productos de seguimiento de graduados y graduadas

\section{Acciones:}

1. Definir los productos.

2. Actualizar la base de personas graduadas.

3. Socializar el portafolio de productos.

\section{Tabla 15}

Medios de verificación del objetivo operativo $S G n .^{\circ} 3$

\begin{tabular}{clc}
\hline \multicolumn{1}{c}{ Indicador } & \multicolumn{1}{c}{ Actores } & \multicolumn{1}{c}{ Meta } \\
\hline Documento portafolio & SGTA & 1 Anual \\
& Extensión Universitaria & \\
& Aseguramiento de la Calidad & \\
& Facultades & \\
& Relaciones Públicas & \\
&
\end{tabular}

Nota. Elaboración propia 
URL: http://www.revistas.una.ac.cr/index.php/dialogo/index

CORREO ELECTRÓNICO: universidadendialogo@una.cr

DOI: http://doi.org/10.15359/udre.11-1.2

Dentro de los medios de verificación se encuentra la presentación anual de un portafolio de productos para las personas graduadas de la Unemi, por parte de los actores involucrados, como se observa en la Tabla 15.

Objetivo operativo n..$^{\circ}$ : Potenciar la bolsa de trabajo - Unemi Empleo Acciones:

1. Adecuar la plataforma informática.

2. Actualizar la base de empresas participantes.

3. Gestionar nuevos convenios para ampliar la oferta de vacantes.

4. Socializar con las personas graduadas y estudiantes.

\section{Tabla 16}

Medios de verificación del objetivo operativo $S G n .^{\circ} 4$

\begin{tabular}{llr}
\hline \multicolumn{1}{c}{ Indicador } & \multicolumn{1}{c}{ Actores } & Meta \\
\hline Estudiantes o personas graduadas que acceden & $\begin{array}{l}\text { Extensión Universitaria } \\
\text { a empleo a través de la bolsa de trabajo. }\end{array}$ & $\begin{array}{l}\text { Relaciones Interinstitucionales } \\
\text { Empresas }\end{array}$ \\
& Empres & \\
\hline
\end{tabular}

Nota. Elaboración propia

Dentro de los medios de verificación se encuentra como indicador que el 10\% de las personas graduadas que interactúa con la bolsa de trabajo debe acceder a fuentes de empleo por medio de los actores involucrados, como se observa en la Tabla 16.

Se observa en la Figura 2 el mapa de procesos de la gestión de vinculación, en donde se establece la relación entre investigación y vinculación, lo cual sirve de entrada para las gestiones de proyectos de vinculación, prácticas pre profesionales, seguimiento de personas graduadas y emprendimientos, que servirán para generar impacto en la sociedad. 
Revista Universidad en Diálogo • Vol. 11, N. ${ }^{\circ}$ 1, Enero-Junio, 2021 • 37-60

\section{Figura 2}

Mapa de procesos de la gestión de vinculación de la Unemi

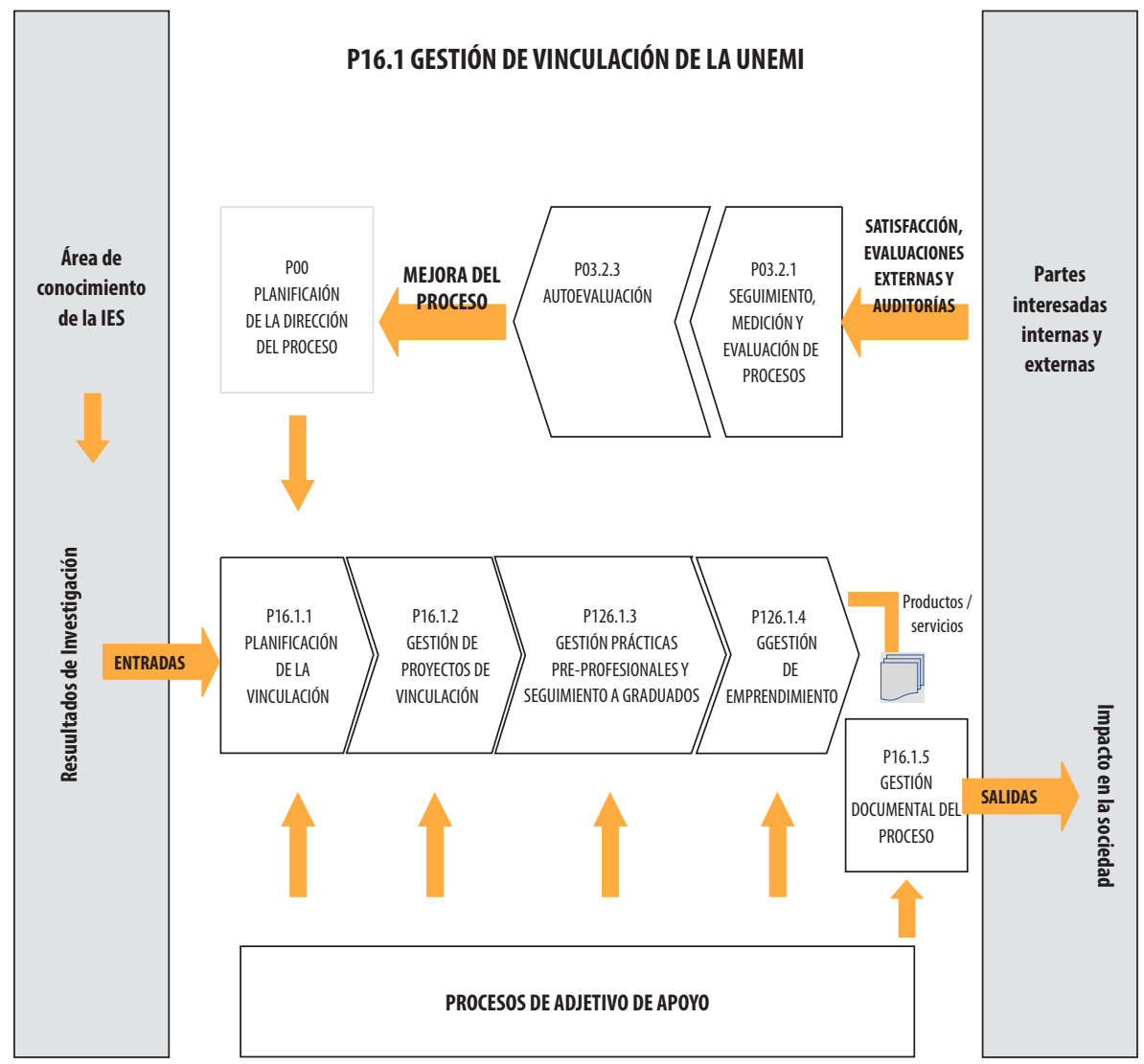

Nota. Elaboración propia

\section{Conclusiones}

A manera de conclusión, se destaca que la Universidad Estatal de Milagro presenta en su planificación estratégica institucional su preocupación y organización de la función sustantiva de la vinculación con la sociedad, para la cual ha creado, acorde con los lineamientos internacionales y las políticas nacionales, un plan que abordará los programas y proyectos de vinculación para ser trabajados por toda la comunidad universitaria. 
Se evidencia la relación que se plantea de la vinculación, tanto con la investigación como con la docencia; con la primera como un soporte de entrada para la generación de proyectos, con lo que, a su vez, al ejecutarse en la vinculación, se retroalimenta a la investigación para nuevos trabajos de campo. Con la docencia de igual manera, ya que este plan estratégico permite a las facultades de la universidad accionarse de manera transdisciplinaria para la ejecución de los proyectos.

\section{Recomendaciones}

Para cumplir con el macro objetivo estratégico y los objetivos operativos resultantes del trabajo realizado en el plan de vinculación con la sociedad al 2021, se recomienda a la universidad:

- Asignar los presupuestos requeridos para la ejecución de los proyectos

- Asignar el talento humano necesario para la correcta ejecución del plan estratégico, del seguimiento y del análisis

- Establecer las relaciones interinstitucionales necesarias para conseguir los stakeholders requeridos

- Involucrar de manera permanente a toda la comunidad universitaria en las actividades de vinculación con la sociedad

\section{Referencias}

Ander-Egg, E. (2013). Introducción a la planificación estratégica. España: Luman Huma.

CES. Reglamento de Régimen Académico. (2017). Ecuador. http://www.ces. gob.ec/lotaip/2017/Diciembre/Anexos Procu/An-lit-a2-Reglamento de Régimen Académico.pdf

D'Este, P., Castro-Martínez, E. y Molas-Gallart, J. (2009). Documento de base para un "Manual de Indicadores de Vinculación de la universidad con el entorno socioeconómico": un marco para la discusión.

Garrocho, C. y Segura, G. A. (2012). La pertinencia social y la investigación científica en la universidad pública mexicana. Ciencia Ergo Sum, 19(1), 26-36. https://cienciaergosum.uaemex.mx/article/view/7770/6344 
Garzon-Castrillón, A. y Zúñiga, X. (2017). Diseño metodológico para la detección de necesidades y elaboración de programas de intervención social. Ciencia Unemi, 10(25), 55-66.

Instituto Nacional de Estadísticas y Censos (INEC). (2012). Proyecciones poblacionales. http://www.ecuadorencifras.gob.ec/ proyecciones-poblacionales/

Instituto Nacional de Estadísticas y Censos (INEC). (2018). Población 2017. http://www.ecuadorencifras.gob.ec/estadisticas/

Red Ecuatoriana Universitaria de Vinculación con la Sociedad (Reuvic). (2012). Propuesta de Plan Nacional de Vinculación de la Educación con la Sociedad. http://www.reuvic.ec/reuvic/index.php?option=com content\&view=article\&id=66:propuesta-de-plan-nacional-de-vinculacion-de-la-educacion-con-la-sociedad\&catid=1:latest-news\&Ite$\underline{\text { mid }=16}$

Universidad Estatal de Milagro. (2018a). Plan Estratégico de Desarrollo Institucional (PEDI) 2018-2021. Ecuador. http://www.unemi.edu.ec/ wp-content/uploads/2018/09/PEDI-2018-2021.pdf

Universidad Estatal de Milagro. (2018b). Plan de Vinculación con la Sociedad al 2021. Ecuador. 